\section{Total and local experimental validation of a lumbar spine numerical model to enhance the orthopaedic management of spinal metastases}

\author{
Simone Borrelli, ${ }^{1}$ Giovanni Putame, \\ Mara Terzini, ${ }^{1}$ Andrea Ferro, ${ }^{2}$ \\ Stefano Marone, ${ }^{2}$ Cristina Bignardi, ${ }^{1}$ \\ Alberto L. Audenino ${ }^{1}$
}

1 PolitoBIOMed Lab, Department of Mechanical and Aerospace Engineering, Politecnico di Torino, Turin; ${ }^{2}$ Oncologic Orthopaedic Surgery Division, CTO

Hospital - Città della Salute e della Scienza di Torino, Turin, Italy

\begin{abstract}
This work shows a full biomechanical validation of a multibody lumbar model with respect to an experimental phantom, fruitful for giving major insights on different surgical vertebral metastasis treatments.
\end{abstract}

\section{Introduction}

The surgical removal of lumbar vertebral body metastasis requires the stabilization of the spine, which is realized, at present, by an extremely invasive procedure consisting in the application of 8 pedicles screws and the fixation of the pathological vertebra with its two superior and inferior adjacent ones. This surgical layout permits a proper biomechanical stabilization at the expenses of spinal mobility, entailing relevant side effects (i.e., overload and rapid degeneration of healthy structural elements of the column, limited range of motions). In this framework, new forms of surgical procedures are being suggested, but their efficacy, in terms of stabilization and load distribution, needs to be proved and verified. Multibody modelling could support the adoption of new solutions predicting their ensuing biomechanical outcomes. Unfortunately, the existing multi-segmental multibody models are mainly validated by recurring to data obtained from single functional units' studies, which do not fully reflect the comprehensive behaviour of multi-vertebral structure. ${ }^{1}$ Hence, this study is intended to provide both a total and local characterization of a validated rachis phantom to permit an exhaustive validation of a complete lumbar in silico model useful to provide robust insights on the biomechanics of novel different surgical layouts designed for spine metastases.

\section{Materials and Methods}

A joint approach, embracing experimental tests and multibody modelling, was then adopted. The experimental protocol consisted in 4 different motion-controlled loads (flexion, extension, compression, and torsion), designed for a linear-torsion test machine (Instron E3000) and applied to a validated Sawbones lumbar spinal phantom (T12 to S1, SKU3430) in its elastic field. The range of motions were limited in order to perform conservative tests and not to overcome the Neutral Zone of the single FSUs. ${ }^{2}$ Relative displacements between vertebrae, postprocessed in GOMcorrelate environment, were also included by the means of planar motion tracking: markers were positioned on the vertebral bodies surfaces and on their spinous processes.

Furthermore, a multibody model was implemented from the Sawbones CAD geometry in MSC Adams. In accordance with the phantom, the passive elements of the spine were included in the model: ligaments were modelled as pre-strained nonlinear tension-only springs, while IVDs were represented as bushing elements oriented coherently with the adjacent vertebrae's endplates..$^{3,4}$ To properly compare the results, the experimental loading protocol was replicated in the numerical environment.

\section{Results}

Starting from data obtained experimentally, the global whole lumbar segment stiff-
Correspondence: Simone Borrelli, PolitoBIOMed Lab, Department of Mechanical and Aerospace Engineering, Politecnico di Torino, Turin, Italy.

E-mail: simone.borrelli@polito.it

Key words: Multibody lumbar model; lumbar model validation; multi-segment spinal biomechanics; sawbones lumbar spine.

Disclosures: None of the authors have any conflicts of interest to disclose.

Conference presentation: This paper was presented at the Third Centro 3R Annual Meeting - L'era delle 3R: modelli in silico, in vitro e in vivo per promuovere la ricerca traslazionale 30 September - 1 October 2021, Evento online organizzato dal Politecnico di Torino.

Received for publication: 9 July 2021.

Accepted for publication: 7 September 2021.

This work is licensed under a Creative Commons Attribution NonCommercial 4.0 License (CC BY-NC 4.0).

(C) Copyright: the Author(s), 2021

Licensee PAGEPress, Italy

Biomedical Science and Engineering 2021; 4(s1):155 doi:10.4081/bse.2021.155

nesses were computed (Table 1). In the investigated ROM, an almost linear behaviour of the dummy was enregistered. Accordingly, the stiffnesses were obtained as the slopes of the angle (T12-L5) - force linear approximation curves $\left(\mathrm{R}^{2}>0.93\right)$.

Concerning the analysis of vertebrae's relative kinematics during the anterior-posterior bending, the angles between the horizontal axis and the lines joining spinous process centers with the vertebral body centre of mas, were measured $(\Delta \phi)$. The multibody model showed a good consistency
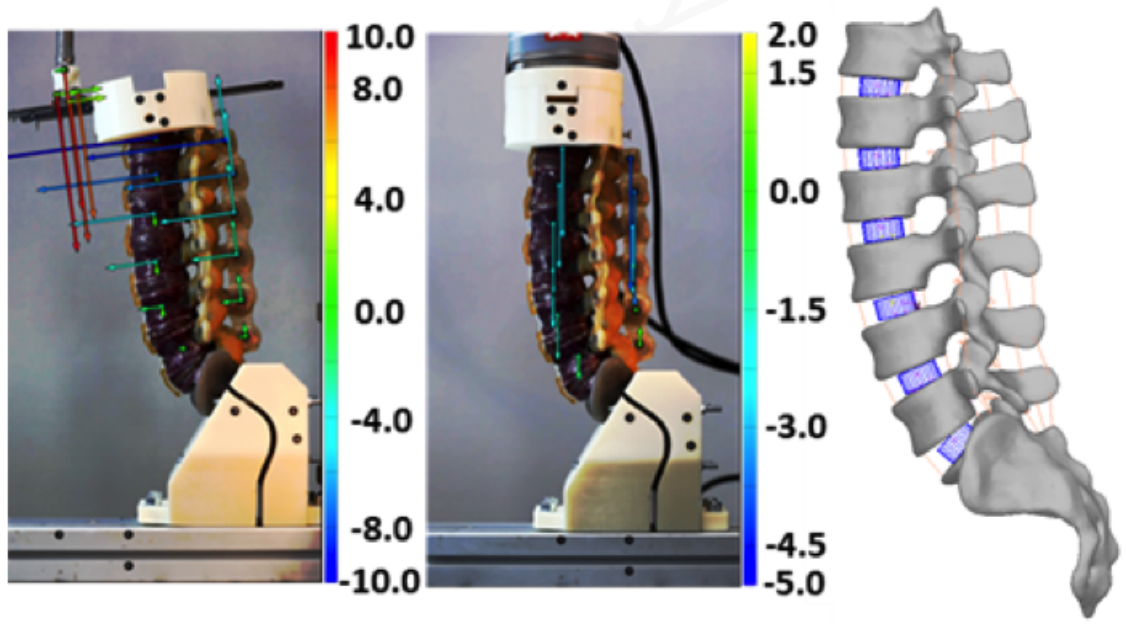

Figure 1. Left and center: experimental setup and kinematic study for the flexion and compression loadings. Right: the lumbar spine multibody model. 
with the experimental results (max discrepancy of 2.5 standard deviation).

Figure 2 reports the case of the anterior bending: although the almost negligible variability of experimental results, the in silico model not only showed the same cranio-caudally decreasing pattern but also close values correspondence.

In the same way, numerical displacements during compression deviate of the $8 \%$ at the maximum amplitude of the experimental motion. Torque was validated only in terms of stiffness since the displacements were out of the sagittal plane.
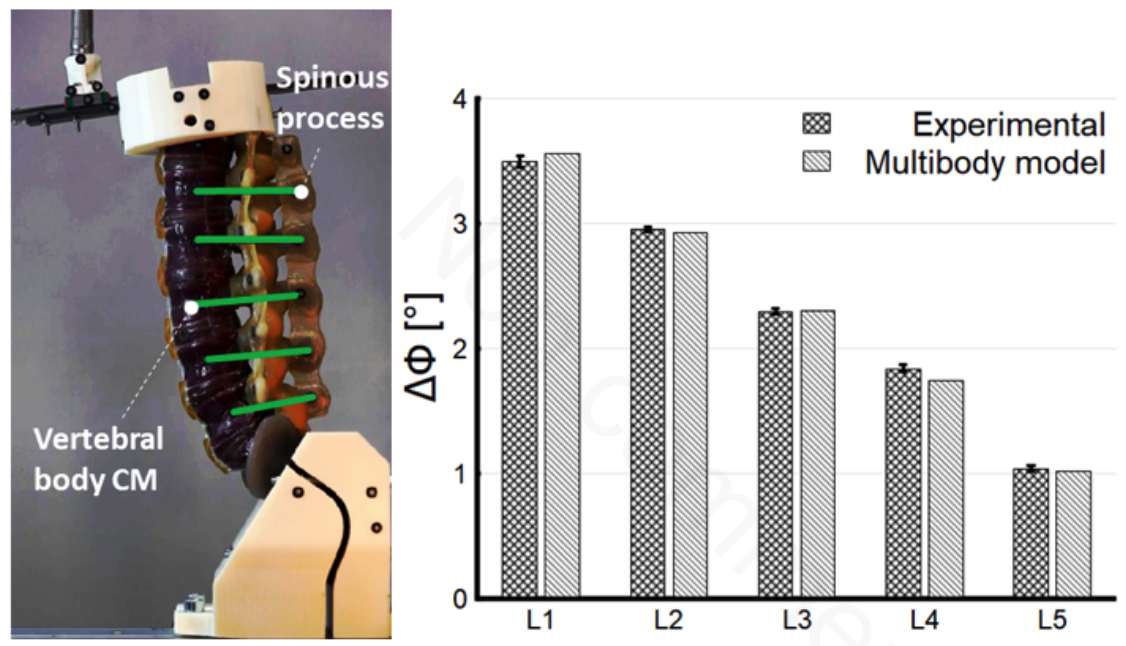

Figure 2. Left: Construction of the vertebral orientation paramenter $\Delta \phi$. Right: Comparison of the experimental and numerical $\Delta \phi$ at the maximum displacement imposed during anterior bending.

Table 1. Results and comparison between experimental ('Exp') and numerical ('Num') lumbar spines' stiffness concerning the global stiffnesses for different motion-loadings. Experimental data reports the mean and standard deviation of 6 replications.

\begin{tabular}{lcc} 
Motion & Exp & Num \\
Flexion $[\mathrm{Nm} / \mathrm{deg}]$ & $1.49 \pm 0.06$ & 1.75 \\
Extension $[\mathrm{Nm} / \mathrm{deg}]$ & $3.45 \pm 0.07$ & 3.03 \\
\hline Compression $[\mathrm{N} / \mathrm{mm}]$ & $94.4 \pm 0.64$ & 87.8 \\
Torque $[\mathrm{Nm} / \mathrm{deg}]$ & $2.7 \pm 0.01$ & 2.8 \\
\hline
\end{tabular}

ing mobility of the column relative to the physiological condition. Those aspects are crucial for the durability of the fixation, the quality of the patients' postoperative life and the risk of implant failure.

Finally, this numerical contribution can support the pre-clinical studies of new orthopaedic fixation devices, pursuing a $3 \mathrm{R}$ approach for a more effective surgical management of spinal metastasis.

\section{References}

1. Zhang C, Mannen EM, Sis HL, et al. Moment-rotation behavior of intervertebral joints in flexion-extension, lateral bending, and axial rotation at all levels of the human spine: A structured review and meta-regression analysis. J Biomech 2020;100:109579.

2. Panjabi MM, Oxland TR, Yamamoto I, Crisco JJ. Mechanical behavior of the human lumbar and lumbosacral spine as shown by three-dimensional load-displacement curves. J Bone Joint Surg Am 1994;76:413-24.

3. Putame G, Terzini M, Bignardi C, et al. Surgical treatments for canine anterior cruciate ligament rupture: assessing functional recovery through multibody comparative analysis. Front Bioeng Biotechnol 2019;7:180. Erratum in: Front Bioeng Biotechnol 2020;8:909.

4. Rupp TK, Ehlers W, Karajan N, et al. A forward dynamics simulation of human lumbar spine flexion predicting the load sharing of intervertebral discs, ligaments, and muscles. Biomech Model Mechanobiol 2015;14:1081-105. 